\title{
"Si hay un riesgo, quiero poder elegir": gestión y percepción del riesgo en los movimientos de reticencia a la vacunación italianos
}

\author{
"If there is a risk, I should have a choice:" Risk \\ management and risk perception in Italian vaccine \\ hesitancy movements
}

'Licenciada en Antropología Médica y Salud Global. Magíster en Antropología Médica y Salud Global, Universitat Rovira i Virgili, Tarragona, Cataluña, España. $\triangle$ iD
RESUMEN En Italia, la aplicación de la obligatoriedad de las vacunas infantiles en 2017 ha sido una consecuencia de la consolidación de la reticencia a la vacunación, un fenómeno que se ha difundido en mayor medida a partir del despliegue de las redes sociales. Este artículo plantea la gestión y percepción del riesgo sobre las vacunaciones infantiles a través del análisis de los contenidos online compartidos por parte de los movimientos de reticencia italianos, realizado entre diciembre de 2019 y abril de 2020. Los resultados señalan que estos movimientos actúan una reinterpretación del riesgo, de la evidencia científica y de la responsabilidad parental sobre todo a través de una presupuesta correlación entre vacuna antisarampión y autismo. Las limitaciones de las decisiones políticas en tema de vacunas se deben a la aplicación de estrategias de carácter punitivo, como sanciones administrativas y exclusión del registro de médicos, que expresan ideas "no vax", factores que pueden aumentar la desconfianza en la clase política y la medicina.

PALABRAS CLAVES Vacuna Antisarampión; Vacunación Obligatoria; Negativa a la Vacunación; Conductas de Riesgo para la Salud; Italia.

\begin{abstract}
In Italy, the passing of mandatory pediatric vaccinations in 2017 was a consequence of increasing vaccine hesitancy in the country, a phenomenon that has largely spread on social networks. This article examines risk management and risk perception regarding pediatric vaccinations through an analysis of online content shared by Italian vaccine hesitancy movements between December of 2019 and April of 2020. Results show that these movements carry out a reinterpretation of risk, scientific evidence, and parental responsibility, especially with regard to the alleged correlation between the measles vaccine and autism. The limitations of political decisions surrounding vaccines are due to the application of punitive measures such as administrative penalties and licensing bans for doctors who express "anti-vax" ideas, aspects which may increase distrust towards the political establishment and the medical profession.
\end{abstract}

KEY WORDS Measles Vaccine; Obligatory Vaccination; Vaccination Refusal; Health Risk Behaviors; Italy. 


\section{INTRODUCCIÓN}

$$
\begin{array}{r}
\text { Es secundario plantearse el problema } \\
\text { de "la" verdad, ya que la verdad } \\
\text { solo es un efecto del poder. } \\
\text { Eduardo Menéndez }{ }^{(1)}
\end{array}
$$

"Mi hijo tenía cinco años y tres meses el día que le inyectaron las vacunas, hablaba, jugaba, hacía todo lo que hacen los niños, también los caprichos, poco después sufrió una regresión y ahora tiene autismo"(2). Así cuenta la madre de Christian en uno de los varios vídeos testimonios del grupo Genitori del No Obbligo Lombardia ${ }^{(3)}$ (padres contra la obligación a la vacunación de la región italiana Lombardía). Christian se vacunó contra sarampión, parotiditis, rubeola, difteritis, tétano, pertussis y poliomielitis el 29 de agosto de 2016. La noche del mismo día sufrió fiebre y se levantó bastante apático y absente el día siguiente. Explica su madre que Christian ya no jugaba ni respondía a los estímulos y, en diciembre, le diagnosticaron autismo.

El grupo de padres contra las vacunas obligatorias de la región Lombardía es una de las diferentes asociaciones italianas que dudan sobre la seguridad y beneficios de las vacunas $\mathrm{o}$, más bien, que consideran dichas vacunas como una técnica peligrosa e invasiva que puede causar enfermedades y síndromes como autismo. Muchos testimonios como los de la madre de Christian empezaron a difundirse en Internet, sobre todo a partir de la introducción de la obligatoriedad a la vacunación infantil por parte del Partido Demócrata, en 2017. La ley, conocida como "Decreto Lorenzin" por el nombre de la Ministra de Salud de entonces, incluye la administración obligada y gratuita de diez vacunas, incluso la vacuna SPR contra sarampión, parotiditis y rubeola (antes eran cuatro). La falta de observancia de la ley conllevará una sanción de 100 a 500 euros $^{(4)}$.

A partir de la introducción de la obligatoriedad, diferentes grupos de padres se reunieron e instituyeron asociaciones a través de las cuales revindicar la objeción de conciencia a las vacunas y compartir contenidos online considerados anticientíficos, por lo cual el pasado gobierno italiano empezó una verdadera "guerra mediática" contra la divulgación de noticias falsas.

Las investigaciones italianas sobre el fenómeno de la reticencia a la vacunación provienen tanto del campo de la salud pública como de la antropología. Desde la perspectiva de la salud pública, un estudio de $2016^{(5)}$ revela que los padres con dudas sobre vacunas serían el $40 \%$ de los encuestados mientras solo el $0,7 \%$ sería propiamente "no vax". Los factores que conllevarían dicha reticencia son: a) el hecho de haber recibido pocas informaciones u opiniones discordantes por parte del propio pediatra; b) conocer a padres que hayan sufrido experiencias negativas con la vacunación; c) utilizar medicinas alternativas. Aunque la cobertura vacunal haya aumentado después de la aplicación de la obligatoriedad ${ }^{(6)}$, resulta que la mayoría de los padres $(60 \%)$ consulta Internet como lugar donde encontrar informaciones sobre las vacunas, por lo que se necesitaría más comunicación en línea por parte de las instituciones científicas, con el objetivo de contrastar las informaciones que proceden de los grupos "antivacunas"(5).

Otra investigación publicada en la revista Vaccinare Oggi e Domani ${ }^{(7)}$ afirma que los grupos de reticencia a la vacunación son bastante variados entre ellos y la difusión de las redes sociales permitió la propagación de sus mensajes, a pesar de que representen una visión extremadamente minoritaria del conjunto poblacional. De forma general, las características principales de dichos movimientos son la creencia de que: a) toda vacuna es peligrosa ya que contiene metales pesados, los cuales son la causa de graves enfermedades como autismo, trastorno de hiperactividad con déficit de atención (THDA) y enfermedades degenerativas y autoinmunes; b) los estudios científicos sobre las vacunas son erróneos puesto que dichos estudios están publicados por las casas farmacéuticas, es decir, los productores de las vacunas que, según las opiniones de estos grupos, solamente están interesados en el provecho económico ${ }^{(7)}$. 
Respecto a las investigaciones del campo antropológico, Oliva(8) analiza la desconfianza entre población y autoridad médicopolítica desde una perspectiva histórica, con un enfoque específico en los factores que conllevaron el nacimiento de los primeros grupos "antivacunas". Por otro lado, lorio(8) conecta el fenómeno de la reticencia con la institución de un nuevo tipo de ciudadanía informada y consciente sobre el propio cuerpo y la salud. En este contexto, cabe destacar la crisis del modelo biomédico y la toma de conciencia de los límites de la medicina occidental a partir de la década de 1970, así como el "revival" de prácticas médicas populares y tradicionales cada vez más requeridas y presentes ${ }^{(9,10)}$ que se inscriben dentro de nuevas corrientes de pensamiento comúnmente definidas como New Age ${ }^{(11)}$.

Importantes aportaciones etnográficas al estudio de la reticencia a la vacunación se deben también a Roberta Raffaetà, antropóloga que condujo una investigación entre familias y profesionales de la salud para entender el proceso de elección a la hora de tomar decisiones en el tema de vacunaciones infantiles y el papel que juegan las asociaciones "antivacunas" en facilitar informaciones y apoyar a aquellos padres que deciden no vacunar a sus hijos. Raffaetà analiza el fenómeno de la reticencia a partir de la reinterpretación del concepto de cuidado por parte de los padres, como medio de autonomía parental para ir en contra de las decisiones políticas ${ }^{(12)}$.

En Italia, sin embargo, hay poco material investigado sobre las comunidades en línea "no vax" y el contenido que comparten. Asimismo, son escasas los estudios antropológicos sobre cómo las instituciones públicas gestionaron el fenómeno de la reticencia a la vacunación y sobre las consecuencias derivadas de la lucha contra la desinformación en línea. Por estos motivos, en este artículo se dará cuenta del fenómeno de la reticencia vacunal italiana a partir de la perspectiva de la antropología médica sobre la gestión del riesgo en materia de salud pública, con un enfoque muy específico de análisis de los contenidos de las páginas web sobre la reticencia a la vacunación. El objetivo es analizar los principales argumentos utilizados por dichos movimientos -sobre todo la relación entre sarampión y autismo-, mostrar cómo la información y la comunicación en las redes sociales puedan influir en la percepción del riesgo y exponer posibles aspectos críticos y limitaciones de las decisiones políticas en el tema de vacunas.

La investigación forma parte de una investigación mayor titulada "'Si hay un riesgo quiero poder elegir': gestión y percepción del riesgo en los movimientos de reticencia a la vacunación italianos" defendida públicamente en septiembre de 2020, en el marco del Máster en Antropología Médica y Salud Global de la Universitat Rovira i Virgili (Tarragona, Cataluña).

\section{METODOLOGÍA}

Tras la cuarentena obligatoria debida a la pandemia de Coronavirus los límites entre "real" y "virtual" ya no se caracterizan por fronteras claras y nítidas, ya que el mundo virtual no solo se ha convertido en un instrumento esencial a través del cual socializar y afrontar las consecuencias de la emergencia sanitaria a nivel existencial, sino también en una herramienta fundamental con la que conducir entrevistas, grupos focales y cuestionarios. Además, el despliegue de las redes sociales ha favorecido la creación y difusión de comunidades en línea y la toma de conciencia de que Internet es un verdadero campo de estudio en que producir datos empíricos ${ }^{(13)} \mathrm{y}$ conducir etnografías del día a día ${ }^{(14)}$.

A partir de estas premisas, he decidido conducir una investigación en línea (online ethnography) sobre los grupos italianos de reticencia a la vacunación, no solo por su relevancia cuantitativa y cualitativa en las redes sociales, sino por el papel que juegan las comunidades virtuales en producir cultura a través de la interacción social, incluso en los procesos de adaptación, utilización de términos simbólicos, reglas, normas sociales ${ }^{(15)}$ y cohesión identitaria. La red social que se ha elegido para conducir la investigación es 
Facebook, y el relevamiento se realizó de diciembre de 2019 hasta abril de 2020.

La primera fase se caracterizó por la búsqueda y la observación previa de los contenidos publicados y los temas más recurrentes sobre reticencia a la vacunación. Se eligieron cuatro páginas públicas para el análisis, ya que creo que el análisis de grupos públicos y privados presupone un problema ético y de difícil gestión. Toda persona que forma parte de un grupo puede publicar cualquier contenido que se ajuste a las normas de la comunidad. Por consiguiente, analizar dichos contenidos presupondría el consentimiento de todos los usuarios, por lo cual opté por focalizarme en los contenidos de páginas públicas, solucionando posibles problemas de ética y privacidad.

Las cuatro páginas se consideraron idóneas por diferentes razones: a) tener un alto número de seguidores; b) tener un alto número de contenido publicado sobre autismo, riesgos de las vacunas y sarampión; c) por ser páginas de Facebook de movimientos políticos; d) por ser páginas de Facebook de asociaciones o personajes importantes. Se envió un correo a las diferentes páginas en el que se especificaron los objetivos y se solicitaron los consentimientos de los administradores. Las páginas analizadas fueron: 1) Associazione Comilva, una de las asociaciones claves en la lucha contra la obligación a la vacunación, fundada en 1993 y que opera en toda Italia a excepción de Trentino-Alto Adigio; 2) Movimento $3 \mathrm{~V}$, movimiento político fundado en 2017 como respuesta a la obligatoriedad de la vacunación; 3) SìAmo, otro movimiento político fundado en 2017 ; 4) VacciniBasta, página informal que no representa ningún tipo de asociación o partido político, pero que se caracteriza por un alto número de seguidores y contenido compartido sobre autismo, sarampión y riesgos de las vacunas. Además, se consideró necesario incluir en el análisis la página de Facebook Medical Facts de Roberto Burioni, médico italiano conocido por su lucha contra los "no-vax" italianos.

Se analizaron aproximadamente 550 contenidos en línea, la mayoría compartidos entre 2015 y 2020 . El análisis se condujo a través de Atlas.ti. en los meses de abril y mayo de 2020. Los datos empíricos se argumentan y se cruzan con las referencias ya existentes en tema de reticencia a la vacunación de investigaciones etnográficas, médicas, históricas y de órganos públicos pertinentes.

\section{Marco teórico y conceptual}

Antes de profundizar el fenómeno de la reticencia a la vacunación italiana cabe destacar dos conceptos esenciales a través de los que se originó el análisis, es decir, el modelo médico hegemónico y la percepción del riesgo.

Con modelo médico hegemónico se entiende aquel sistema de pensamiento, construcción de la realidad y concepción de las enfermedades sobre los cuales la medicina occidental se instituyó como el sistema médico más utilizado a nivel mundial. Según Menéndez ${ }^{(16)}$, el modelo médico hegemónico se define, entre otros, por su ahistoricidad, su relación asimétrica entre médico y paciente, su individualismo y su legitimización a través del proceso de medicalización, de la sociedad $^{(17)}$. Sin embargo, hablar de hegemonía presupone la existencia de otras prácticas a las que los individuos recurren en caso de enfermarse: medicamentos populares, en la actualidad sobre todo alternativos y complementarios, que siempre han jugado un papel fundamental en las elecciones terapéuticas y que Menéndez define a través del modelo alternativo subordinado y de la autoatención.

El modelo alternativo subordinado se delimita por su concepción globalizadora de la enfermedad, aunque estas prácticas sean derivaciones del modelo médico hegemónico: por este motivo, se cargan de valores del pasado caracterizándolos por acepciones biomédicas, es decir, ahistoricidad, individualismo y asimetría en las relaciones, características bien representadas por los movimientos New Age.

Por el contrario, el modelo de autoatención se determina como el primer nivel real de atención médica llevado a cabo por toda persona y grupo parental, y se define como 
un modelo incorporado a través del que gestionar y prevenir el riesgo.

Ahora bien, en nuestra sociedad actual, el riesgo se determina como una manera de construir la realidad que encuentra su significado más profundo en la definición de "sociedad del riesgo" planteada por Beck. La modernidad se caracteriza por ser una condición existencial delimitada por la imposibilidad de escaparse de riesgos y peligros ${ }^{(18)}$. Según Sepúlveda(19), el riesgo parece ser un resultado cierto a corto, mediano o largo plazo, que se inscribe dentro de una nueva ecuación "riesgo-peligro-daño". El riesgo representaría entonces una manera moderna de percibir y gestionar el peligro, ya sea real o imaginario ${ }^{(20)}$; peligro que juega un papel fundamental en las prácticas de prevención de salud pública, como en el caso del Coronavirus o la vacunación en general. Por este motivo, el riesgo no solo es una específica manera de construir y percibir la realidad, sino también una forma objetiva de racionalizar los eventos con el fin de hacerlos gobernables ${ }^{(21)}$.

Dice Lupton ${ }^{(22)}$, el modelo médico hegemónico suele representar el riesgo según una visión estrictamente individualista. En nuestra sociedad, los riesgos en salud se caracterizan por ser un fenómeno que depende de la responsabilidad de los individuos: estar saludable representa la condición primaria a través de la cual evitar riesgos para el bienestar de uno mismo y el conjunto poblacional. A pesar de que las estrategias de prevención se gestionen mediante cálculos y estadísticas, los discursos sobre el riesgo detentan una función altamente política y moralizadora que definen a sujetos "en riesgo" y sujetos "de riesgo"(22). Parafraseando a Douglas ${ }^{(23,24)}$, los discursos sobre el riesgo se determinan como una estrategia a través de la cual vigilar y controlar al conjunto poblacional. La retórica utilizada apela a las emociones.

El miedo y el sentido de culpabilidad son herramientas por medio de las cuales culpabilizar a los sujetos "de riesgo", es decir, los sujetos irresponsables, y mantener la cohesión social del grupo. Ahora bien, como afirma Romaní "uno de los vehículos privilegiados de expansión del miedo en nuestras heterogéneas sociedades es el de los medios de comunicación social"(25). Dicha expansión del miedo ha conllevado un exceso de información sobre los riesgos, que se caracteriza por la disminución de la confianza en el Estado y la ciencia, incluso la necesidad de tomar el control sobre la propia vida ${ }^{(26)}$. En la era de las redes sociales los discursos sobre el riesgo se caracterizan por una manipulación y reinterpretación de la retórica institucional a través de las cuales los movimientos de reticencia a la vacunación italianos han ido definiendo nuevos sentidos del riesgo bajo una nueva ecuación de sujetos en riesgo, sujetos de riesgo y técnicas arriesgadas.

\section{RESULTADOS}

\section{La nocividad de las vacunas: la correlación entre autismo y vacuna contra el sarampión}

La correlación entre autismo y vacuna antisarampión es el asunto principal de un estudio inglés, publicado por The Lancet, en 1998, en que el médico gastroenterólogo Andrew Wakefield afirmó un posible vínculo entre la vacuna contra el sarampión (es decir, la vacuna triple vírica contra sarampión, rubeola y parotiditis) y dicho síndrome ${ }^{(27)}$. El estudio suscitó un acalorado debate en la comunidad científica hasta que Wakefield estuvo bajo investigación por falta de ética profesional y mala praxis, por lo cual fue excluido del registro médico en 2010, no obstante, la relación entre autismo y vacuna sigue teniendo bastante éxito dentro de los movimientos de reticencia a la vacunación a pesar de haberse demostrado posteriormente que tal relación no existe.

Ahora bien, aunque Wakefield fue excluido del registro médico, sigue siendo unos de los líderes más importantes de los movimientos "no vax".

[Pedimos] jLa rehabilitación total de A. Wakefield ridiculizado y marginado por parte de toda la comunidad científica 
denominada de bien, ya que sus descubrimientos y estudios perturbaron las teorías fantasiosas según las cuales no existiría ninguna correlación entre autismo y vacunas! ¡Reflexionar atentamente, informarse y aprender a usar la cabeza si se decide a vacunarse o si hay necesidad absoluta de hacerlo, esto es esencial para evitar posibles reacciones adversas, incluso letales, en nuestros hijos! [(Chiediamo) La totale riabilitazione di A. Wakefield deriso, schernito ed emarginato da tutta la comunità scientifica cosiddetta per bene, perché le sue scoperte e $i$ suoi studi hanno sconvolto le teorie fantasiose secondo le quali non vi sarebbe nessuna correlazione fra autismo e vaccini! Riflettere bene, informarsi e ragionare con la propria testa se proprio si decide di vaccinare o vi è la assoluta necessità di farlo, è la prima regola per evitare possibili reazioni avverse anche letali sui nostri figli!] (Página de Facebook VacciniBasta) ${ }^{(28)}$

Wakefield ha llegado a dirigir dos documentales, muy conocidos en Italia, sobre el vínculo sarampión-autismo que se titulan "Vaxxed" (2016) y "Vaxxed II: The People's Truth" (2019). Además, la utilización de fuentes estadounidenses es bien elevada dentro de los grupos de reticencia que analicé a excepción del Movimento 3V.

Los fundamentos principales de las teorías que vinculan autismo y vacuna contra el sarampión se pueden detectar, sobre todo, en la página de Facebook y en el sitio web de la Associazione Comilva, que dice:

Las vacunas causan daño gastrointestinal [...]. Y esto lleva al diagnóstico definitivo de "autismo". Si las observaciones del doctor Wakefield están correctas, al fin y al cabo alguien, en algún lugar, conectará los puntos y llegará a la correlación entre vacunas y el efecto que conlleva el diagnóstico de autismo. [I vaccini causano danno gastrointestinale [...] Ed è questo che porta alla diagnosi definitiva di "autismo". Se le osservazioni del dott.
Wakefield sono corrette, qualcuno, da qualche parte, alla fine unirà i puntini e arriverà alla connessione tra vaccini e I'effetto domino che porta alla diagnosi di autismo]. (Página web Associazione Comilva) ${ }^{(29)}$

El autismo es un síndrome del que, en parte, se desconocen las causas. Según la Associazione Nazionale di Genitori Soggetti Autistici (ANGSA), solo se descubrieron las causas biológicas que influyen en el desarrollo cerebral del infante. Además, en Italia, las informaciones sobre personas con autismo son escasas y poco homogéneas, ya que no existen datos públicos sobre el número de sujetos afectados. En la actualidad, el índice es de 4 de cada mil niños; sin embargo, la asociación declara que se trata de un trastorno de difícil diagnóstico, sobre todo con respecto a los casos menos graves ${ }^{(30)}$. Ahora bien, el hecho de que el autismo sea un síndrome aún poco conocido podría ser una información relevante para entender la visión que tienen los movimientos de reticencia con respecto a dicho trastorno y su correlación con la vacunación, ya que los primeros síntomas se manifiestan generalmente en el mismo período en que se suministran las vacunas infantiles. Por esto, la Associazione Comilva expresa que "la edad mejor para vacunar es después de los 18 meses, ya que la mayoría de los eventos adversos se verifican en gran medida antes de los 2 años" [L'età vaccinale migliore è dopo i 18 mesi, perché la maggior parte degli eventi avversi si verificano in massima parte prima dei 2 anni $]^{(31)}$.

Según los movimientos de reticencia, el motivo por el que dichas vacunas podrían conllevar enfermedades graves ha de entenderse dentro de la creencia de que las vacunaciones no son seguras ni inocuas, ya que contienen elevadas cantidades de metales pesados como aluminio, mercurio, formaldehído y ADN fetal.

En el conjunto de vacunas analizado emerge que, entre adyuvantes, excipientes y contaminantes se observa la presencia de 14 componentes tóxicos o 
potencialmente tóxicos y 22 componentes causantes de reacciones alérgicas, sin mencionar los contaminantes biológicos -es decir, virus, bacterias, micoplasma y micobacterias, así como ADN fetal humano y ADN animal- que provienen de tejidos biológicos de cultivo de las vacunas. [Nell'insieme dei vaccini analizzati emerge che fra adiuvanti, eccipienti e contaminanti si registra la presenza di 14 componenti tossici o potenzialmente tossici e 22 componenti scatenanti fenomeni allergici, senza contare i contaminanti biologici - ovvero virus, batteri, micoplasmi e micobatteri, nonché DNA umano fetale e DNA animale - che provengono dai tessuti biologici di coltura dei vaccini]. (Página web Associazione Comilva) $)^{(32)}$

Además, aunque la medicina afirme que los efectos colaterales de las vacunas son una consecuencia normal de la inyección y que el riesgo de problemas graves es bajo o nulo, estos movimientos tienen una elevada percepción del riesgo por los efectos colaterales de la vacunación.

Las vacunas causan condiciones físicas que llevan al dolor, al daño neurológico, a los desórdenes del sistema inmunitario, al daño gastrointestinal, y al desarrollo excesivo de candidiasis, todos factores que si son combinados entre ellos producen síntomas comportamentales que llevan al diagnóstico de "autismo". [l vaccini causano le condizioni fisiche che portano al dolore, al danno neurologico, ai disordini del sistema immunitario, al danno gastrointestinale, ed alla crescita eccessiva di candida - tutte cose che combinate producono i sintomi comportamentali che portano alla diagnosi di "autismo"] (Página web Associazione Comilva) ${ }^{(33)}$

En la base de sus preocupaciones, la vacuna se percibe como un elemento "externo" que se inyecta en infantes "sanos", una experiencia negativa, tanto para los niños como para los padres, que no se considera necesaria, ya que la mayoría de las enfermedades por las que se vacuna ya no existen o no se perciben como problemáticas.

Por lo que se refiere al sarampión, Italia recomendó la vacuna triple vírica sarampiónrubeola-parotiditis en $1999^{(34)}$, mientras su obligación se introdujo en 2017 por primera vez como consecuencia de los brotes. En biomedicina, el concepto clave para lograr la eliminación del sarampión es mantener una cobertura del $95 \%$ a través de dos dosis de vacuna triple vírica. Según el último informe de la comisión europea de la Organización Mundial de la Salud (OMS), Italia se sitúa entre los países con transmisión endémica de sarampión, es decir, que afecta de forma permanente el país, con tasas de incidencia de 88,4 por millón de habitantes, habiéndose notificado 5.404 casos con 4 muertes y un total de 634 brotes en las diferentes regiones del país en 2017. La cobertura vacunal con dos dosis de la triple vírica estaba en ese mismo año en torno al $86 \%$, y resulta que el motivo de dicha disminución es consecuencia de la pérdida de confianza en las instituciones médico-sanitarias ${ }^{(35,36)}$.

Es cierto que los grupos "antivacunas" no se crearon en este período histórico: resulta que el primer movimiento de oposición se instituyó en 1853 en Inglaterra, en correlación con el Vaccination Act del mismo año, un plan de vacunación que establecía la obligación a vacunarse ${ }^{(37)}$; sin embargo, la correlación entre autismo y vacuna antisarampión favoreció un "revival" de estas creencias por lo cual las tasas de vacunación decrecieron en EEUU y muchos países europeos, entre ellos, Italia ${ }^{(38)}$.

Ahora bien, la mayoría de las personas adultas italianas no se vacunó o más bien sufrió la enfermedad, y tal vez esto pueda representar uno de los motivos principales por los que los movimientos de reticencia tiendan a percibir el sarampión como una enfermedad benigna.

En época prevacuna, cuando el virus del sarampión natural infectaba a toda la población, el sarampión -"típicamente 
una enfermedad infantil benigna", afirmaba Clinical Pediatrics- se veía como una enfermedad que facilitaba la inmunidad de por vida, evitando así infecciones peligrosas para los adultos. [In epoca pre-vaccino, quando il virus del morbillo naturale infettava tutta la popolazione, il morbillo - "tipicamente una malattia infantile benigna", come Clinical Pediatrics descriveva - veniva accolto per la fornitura dell'immunità a vita, evitando così infezioni pericolose per gli adulti]. (Página web Associazione Comilva) $^{(39)}$

Además, se suelen banalizar los brotes de sarampión hasta considerarlos una estrategia mediática y política para obligar a la población a vacunarse:

La nueva documentación sobre el sarampión ha salido... ¿Qué podríamos decirles a todos aquellos mentirosos, políticos y no, que utilizaron esta falsa epidemia como piedra angular para la obligatoriedad de la vacunación? Lorenzin, una vez que las mentiras se descubren la gente exige explicaciones y motivos reales de la existencia de este decreto. [Ė uscito il nuovo bollettino del morbillo... cosa si può dire a tutti quei menzogneri, politicanti e non, che hanno utilizzato come portabandiera per lıbbligo vaccinale questa finta epidemia? Lorenzin una volta che le bugie vengono a galla la gente esige spiegazioni e motivazioni reali per questo decreto] (Página de Facebook VacciniBasta) ${ }^{(40)}$

La autoridad médica y política a menudo se representa como un poder oculto y estafador: el acento se pone en la corrupción de un gobierno sometido a las decisiones de las casas farmacéuticas y de médicos cuyos intereses van en contra del bienestar de la población:

"Claramente no fue culpa de la vacuna". ¡No! ¡Claro que no! Esto es lo que dicen las celebridades, y claro que es así. Como si no viviéramos en un mundo corrupto en el que los medias influenciarían la opinión pública y las casas farmacéuticas corromperían a magistrados, investigadores, médicos. [...] Vosotros que negáis la evidencia, que no tenéis dudas [...] traed vuestras buenas intenciones a mirar el mar. Tal vez uno reconozca el horizonte y entienda que los terraplanistas sí sois vosotros. ["Certamente non è stato il vaccino". No! Categoricamente no! Questo dicono i luminari, per cui è certamente così. Mica viviamo in un mondo corrotto in cui $i$ media influenzano l'opinione pubblica e le case farmaceutiche possono corrompere magistrati, ricercatori, medici. [...] Voi che negate l'evidenza, che non avete dubbi [...] portate $i$ vostri buoni propositi a guardare il mare. Notereste l'orizzonte e probabilmente capireste che i terrapiattisti siete proprio voi.] (Página de Facebook SìAmo) $)^{(41)}$

Además, se cree que las vacunas no siempre crean inmunidad, es decir, que la inmunidad al $100 \%$ es una utopía o no está garantizada de por vida, ya que producen inmunidad artificial:
La vacuna contra el sarampión no crea una inmunidad permanente. Las vacunas solamente confieren una inmunidad arti- ficial temporánea y, a veces, ni siquiera la alcanzan (sujetos no respondedores): esto es el motivo por el que los profe- sionales de sanidad recomiendan dosis múltiples para aumentar la inmunidad adquirida artificialmente. [II vaccino contro il morbillo non crea un'immunità permanente. I Vaccini conferiscono solo immunità artificiale temporanea e a volte non ci riescono nemmeno (soggetti non responder): questo è il motivo per cui i funzionari della sanità pubblica racco- mandano dosi multiple di questo vac- cino per aumentare l'immunità artificiale acquisita.] (Página web Associazione Comilva) ${ }^{(42)}$

Sin embargo, a pesar de que estos movimientos piden que se quite la obligatoriedad de la vacunación, no todos están totalmente en contra de la utilidad de las vacunas en sí, 
ya que el problema se sitúa en la elevada cantidad de metales pesados, hecho que se podría resolver a través de investigaciones independientes sobre vacunas que puedan disminuir su toxicidad, y en el uso de una medicina personalizada. Ahora bien, las peticiones fundamentales de estos movimientos son básicamente dos, es decir, la libertad de elección y el principio de precaución en el tema de vacunas.

Lo que subrayan estos movimientos es que la edad de los niños es demasiado precoz para vacunarlos y que la obligatoriedad de diez vacunas es irracional e infructuosa, una decisión política considerada agresiva y que no tiene nada a que ver con el uso de una medicina que apunte al bienestar $y$ al beneficio. Por este motivo, dichos movimientos proponen la implementación de pruebas prevacunación y vacunas monodosis a través de las cuales se pueda evitar la aplicación de cantidades elevadas de metales pesados y, en definitiva, una prevención más personalizada. Además, el tema de las vacunas monodosis fue propuesto también por Matteo Salvini, hecho que revela cómo la reticencia a la vacunación no es un fenómeno meramente "popular", ya que se convirtió en un fenómeno político aparentemente apoyado por Salvini y el Movimento 5 Stelle, que empezaron unas campañas políticas contra la ley de obligatoriedad de la vacunación del Partido Demócrata.

Matteo Salvini dice en su página de Facebook:

El gobierno obligado a copiar y aprobar nuestra propuesta para las vacunas monodosis. Continuar así: siempre del lado de los padres, de los niños, de la libertad terapéutica sin restricciones. Basta con este Gobierno! \#bastaPD [Governo costretto a copiare e approvare la nostra proposta per $i$ vaccini monodose. Avanti così: sempre dalla parte dei genitori, dei bambini, della libertà di curarsi senza imposizioni. Governo a casa! \#bastaPD] (Página de Facebook Matteo Salvini) ${ }^{(43)}$
Asimismo, escribe el Movimento 5 Stelle:

Consideramos las vacunas fundamentales en la prevención de las enfermedades y nuestro objetivo es garantizar la máxima cobertura vacunal en nuestro País. ¿Cómo alcanzar este objetivo? El decreto Lorenzin se basa en la coacción, y se expone al riesgo peligrosísimo de obtener el efecto contrario. [Riteniamo $i$ vaccini fondamentali nella prevenzione delle malattie e il nostro obiettivo è garantire la massima copertura vaccinale nel Paese. Come raggiungere questo obiettivo? I/ decreto Lorenzin punta tutto sulla coercizione, esponendosi al rischio, pericolosissimo, di ottenere l'effetto contrario.] (Página de Facebook Movimento 5 Stelle) $)^{(44)}$

Subrayo que dicho apoyo fue aparente, ya que cuando la Liga y el Movimento 5 SteIle ganaron las elecciones en 2018 no consiguieron encontrar un acuerdo político en el tema de vacunas apoyado por todos los parlamentarios, por lo que la obligatoriedad de la vacunación sigue siendo aplicada. Por otro lado, los grupos de reticencia instituyeron movimientos políticos propios, como el SìAmo y el Movimento 3V, en 2017. Este último participó a las elecciones regionales de Emilia-Romaña en enero de $2020^{(45)}$. La batalla mediática entre partidos, movimientos políticos, médicos y ciudadanos, entre quienes apoyan el actual discurso biomédico hegemónico y quienes lo rechazan, se caracteriza indudablemente por una polarización de la realidad y un endurecimiento de ambas posiciones. Dice el Movimento 3V:

El enemigo es quien impuso la obligación de la vacunación y quien, una vez en el poder, no la quitó. [Ritiene che il proprio nemico sia soltanto chi ha imposto l'obbligo e chi, una volta al governo, non lo ha tolto.] (Movimento 3V Vaccini Vogliamo Verità)(46)

Ahora bien, como se ha dicho anteriormente, la figura del médico Roberto Burioni juega 
un papel fundamental en la "lucha" contra los movimientos de reticencia a la vacunación, hecho por el que sus discursos se analizarán a continuación.

\section{Batallas mediáticas: la lucha contra la mala información}

La página de Facebook Medical Facts de Roberto Burioni se creó en 2016 con el objetivo de establecer una relación de confianza con todo aquel público que aún no podía contar con un referente oficial en las redes sociales, a través del cual resolver las diferentes dudas sobre las vacunas.

La creación de Medical Facts se debe, desde luego, a una necesidad detectada originalmente en la falta de una comunicación médica que pudiese instituir un vínculo entre biomedicina y población, así como entre biomedicina y un tipo de divulgación virtualmente accesible a los demás. Como afirma el propio Burioni, su página de Facebook nace de la necesidad de dar a entender que "en medicina cuentan los hechos, no las opiniones" ["in medicina contano i fatti, non le opinioni $^{(147)}$, subrayando así la urgencia de restablecer una frontera entre conocimientos biomédicos y discursos meramente "populares" que no tienen en consideración la evidencia científica y la verificación de los datos en el tema de vacunas, sino que basan sus conocimientos y teorías en noticias falsas cuya presencia en la web se determina como un factor preponderante en el crecimiento de la reticencia a la vacunación.

La difusión de mentiras peligrosísimas, sobre todo cuando se habla de salud, es un fenómeno gravísimo. Debido a estas mentiras muchas personas toman malas decisiones, no se curan, no vacunan a sus hijos. La consecuencia es la muerte de algunas personas. [La diffusione di bugie pericolose, soprattutto quando si parla di salute, è una cosa gravissima. A causa di queste bugie molte persone fanno scelte sbagliate, non si curano, non vaccinano i figli. La conseguenza di questo è la morte di alcune persone.] (Página de Facebook Medical Facts) ${ }^{(48)}$

El tipo de comunicación que Burioni ha elegido no se destaca de manera tan nítida de aquella de los movimientos denominados "no vax", es decir, utiliza un lenguaje bastante persuasivo y práctico con el objetivo de explicar y convencer a sus seguidores de las fallas cognitivas en la que se funda la retórica sobre la nocividad de las vacunas. Ahora bien, dice Burioni:

\begin{abstract}
La ciencia necesita una nueva voz que no se encuentra en el lenguaje de los congresos científicos, sino en un lenguaje comprensible, apasionado y convincente. [La scienza ha bisogno di una nuova voce che non si incontra nei congressi scientifici ma in un linguaggio comprensibile, appassionato e convincente. $]^{(49)}$
\end{abstract}

El uso de datos científicos e institucionales se cruza con un tipo de comunicación bastante directa, es decir, que representa a los pertenecientes de los movimientos de reticencia como personas que ponen en peligro el bienestar del conjunto poblacional y que minan el fundamento de los principios científicos a través de creencias supersticiosas sobre la nocividad de las vacunas. La reticencia se representa así como un daño colectivo mediante el cual identificar nuevos sujetos de riesgo y sujetos en riesgo.

A los primeros se asocian aquellas personas que confían en noticias falsas y artículos pseudocientíficos en lugar de consultar al propio médico de cabecera en el tema de vacunas, mientras que con "sujetos en riesgo" se define todo el conjunto poblacional que, a raíz de la reticencia, no se puede beneficiar de la protección de la cobertura vacunal establecida al 98\%. El eje principal de los discursos de Burioni se caracteriza por la apelación al sentido común: el médico afirma que las creencias de dichos movimientos proceden de una profunda ignorancia sobre las vacunas que a menudo se compara con una visión bastante oscurantista y medieval de la percepción de la realidad. 
Tenemos que tutelar el derecho a la salud y la educación de aquellos niños [inmunodeprimidos] que no pueden vacunarse, y no la pretensión de padres irresponsables y obscurantistas que ponen en peligro la vida de sus hijos en nombre de supersticiones sin sentido. [Dobbiamo tutelare il diritto alla salute e alla istruzione dei bambini che non si possono vaccinare, non la pretesa dei genitori irresponsabili e oscurantisti di mettere a rischio la vita dei loro figli in nome di superstizioni senza senso.] (Página de Facebook Medical Facts) ${ }^{(50)}$

Los reticentes se presentan como personas a veces violentas cuyo objetivo es generar miedo y peligro a través de sus mensajes irracionales. Subraya Burioni que los estudios científicos no han detectado ninguna correlación entre autismo y vacuna, y que el sarampión es una enfermedad grave que puede conllevar la muerte. Frente a los enormes gastos en salud debidos a brotes evitables, la vacuna representa una técnica segura y eficaz que implica poca inversión económica, cuyas reacciones adversas son casi inexistentes e inofensivas.

En definitiva, no solo los "no vax" representan un peligro para la difusión de brotes de enfermedades graves que se podrían evitar a través de la vacunación, sino también para todos aquellos niños inmunodeprimidos que no pueden vacunarse, inclusive para sus propios hijos.

La ciencia nos dice con certidumbre que las vacunas son seguras y eficaces, y que si la tendencia actual de no vacunar a los hijos no cambia, las consecuencias graves no afectarán solamente a los no vacunados, sino que involucrarán a toda la sociedad. [...] En todo caso, es bueno recordar que - si se decide de recorrer la vía de la persuasión, la que adoran los contrarios a la obligación - será necesario persuadir de la eficacia y utilidad de las vacunas aquellas personas convencidas que el sarampión (una enfermedad que, recuerdo a todos, es muy seria) no se puede curar eficazmente vistiendo a los niños de rojo [práctica popular italiana]. [La scienza ci dice con certezza che $i$ vaccini sono sicuri ed efficaci, e che se la attuale tendenza a non vaccinare i figli non sarà invertita andremo incontro a gravi conseguenze che non si limiteranno a danneggiare $i$ non vaccinati, ma coinvolgeranno tutta la società. [...] In ogni caso è bene ricordare che - se si decide di percorrere la strada della persuasione, quella che amano i contrari all'obbligo sarà necessario persuadere dell'efficacia e dell'utilità dei vaccini persone convinte che il morbillo (una malattia che, vi ricordo, è molto seria) si può curare efficacemente vestendo i bambini di rosso.] (Página de Facebook Medical Facts) ${ }^{(51)}$

Dice Burioni, la obligación de vacunarse se representa como una respuesta útil y necesaria debida a la irresponsabilidad de personas reticentes, sin embargo, según mi análisis, la obligación se determina como un proceso de normativización a través del que restablecer una relación de poder jerárquico entre Estado, institución médica y sociedad. Subrayo que dicho proceso de normativización no solo se ha instituido a través de la emisión de sanciones administrativas, sino más bien a través de la exclusión del registro de todos aqueIlos médicos que expresan ideas antivacunas como Roberto Gava ${ }^{(52)}$ y Dario Miedico(53), personaje importante de la Associazione Comilva y cofundador del movimiento político SìAmo, mientras que la invisibilización online de los grupos de reticencia a la vacunación por parte de Facebook ${ }^{(54)}$ representa un fenómeno adicional a la lucha contra la desinformación que se ha aplicado también con el Coronavirus.

Además, dicha normativización se ha reforzado a través del apoyo político a personajes "pro vax" como Burioni, de una narrativa "a favor de la ciencia" por parte del Partido Demócrata (Figura 1) y de campañas de prevención en tema de vacunas bien persuasivas que alimentan un sentimiento de vulnerabilidad al igual que la retórica utilizada por los grupos de reticencia (Figura 2 y Figura 3). 


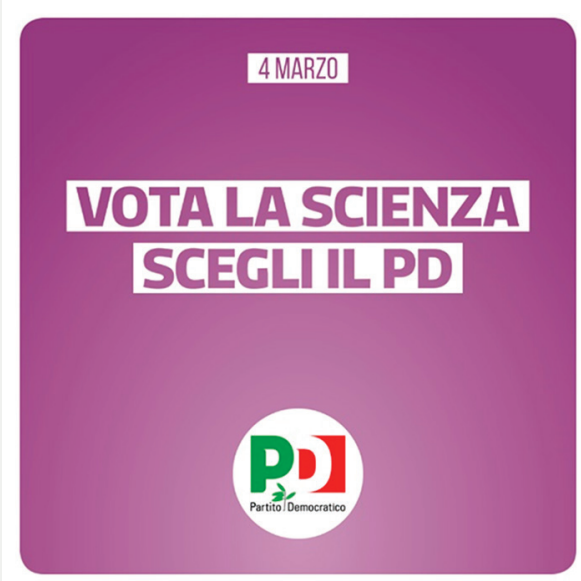

Figura 1. "Vota por la ciencia, vota por Partido Demócrata". Roma, Italia, 2018.

Fuente: Partido Democratico.

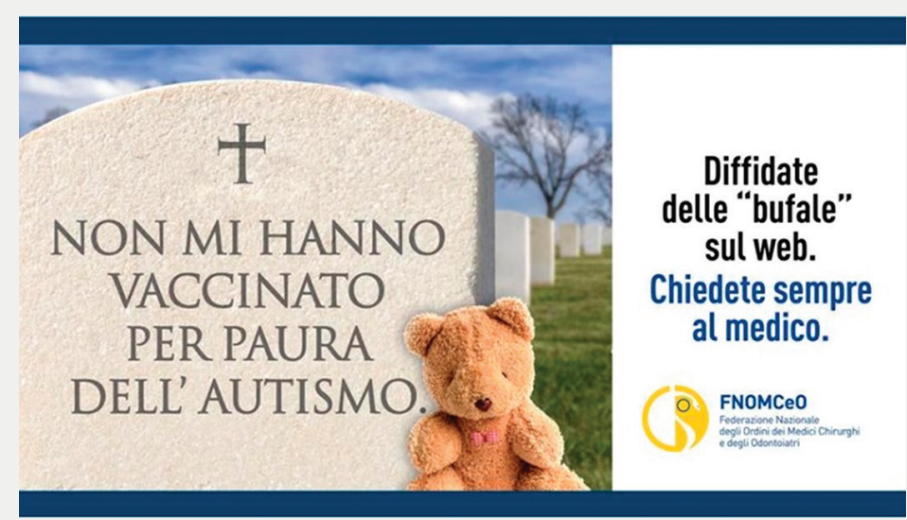

Figura 2. "No me vacunaron por miedo al autismo". Roma, Italia, 2018. Fuente: Federazione Nazionale degli Ordini dei Medici Chirurghi e degli Odontoiatri.

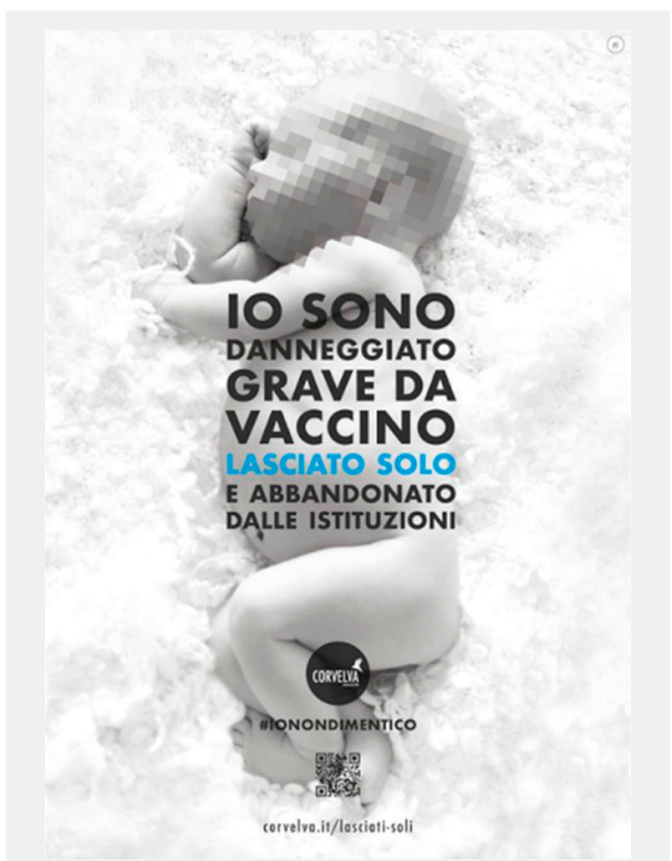

Figura 3. "Soy un afectado grave por vacuna, dejado solo y abandonado por las instituciones". Vicenza, Italia, 2017..

\section{DISCUSIÓN}

"No hay tal cosa como la sociedad. Hay hombres y mujeres y hay familias" afirmó Margaret Thatcher en su célebre discurso del 1987. En aquellos años, la caída de los Estados de bienestar europeos y políticas públicas adecuadas conllevó la institución del neoliberalismo: en los ciudadanos recaía la responsabilidad individual de su vida y su situación económica y social. "No hay Estado, hay familias" es lo que afirman los movimientos de reticencia a la vacunación italianos. Las narrativas analizadas se caracterizan indudablemente por una reconfiguración del vínculo entre institución, sociedad e individuo y por una interpretación del riesgo que se determina a través de la creencia de que la vacuna es un método de prevención inseguro y dañino. Como la vacunación representaría uno de los primeros actos médicos que se aplica al conjunto poblacional, en estos grupos destaca una tendencia que surge de la necesidad de reivindicar la autonomía individual y legitimidad como padres ${ }^{(55)}$.

Lo que reclaman estos grupos es la exigencia de adquirir nuevos derechos sociales que se conectan con la patria potestad y libertad en el tema de elección terapéutica, exigencia que juega un papel fundamental en la institución de nuevas ideologías y figuras parentales, estas últimas caracterizadas por la ultra información, es decir, la tendencia a informarse de manera elevada tanto a través de datos institucionales como informales, y una atención excesiva hacia la progenie.

Criar al propio hijo sano y saludable es la justa preocupación de cada padre, sobre todo hoy en día, ya que debemos ser cada vez más responsables y atentos de su camino evolutivo. [Crescere il proprio figlio sano e in buona salute è la giusta preoccupazione di ogni genitore, soprattutto al giorno d'oggi che sei chiamato a diventare sempre più partecipe e responsabile del suo percorso evolutivo.] (Página de Facebook Associazione Comilva) ${ }^{(56)}$ 
No solo la reticencia vacunal es un fenómeno que se conecta con la reinterpretación de los riesgos modernos y la evidencia científica, sino que conlleva también una manipulación del concepto de responsabilidad, entendida como un deber individual que se asocia a la idea de un Estado estafador incapaz de proteger a sus ciudadanos. Por este motivo, los padres son los únicos que pueden satisfacer las exigencias de sus hijos y cuidarlos de manera correcta.

Ayer el Estado abandonó deliberadamente a los padres que han elegido conscientemente por la salud de sus propios hijos, proponiendo un decreto que apunta a la marginación y el empobrecimiento de las familias, utilizando epidemias falsas, chantajes y llamadas al orden. Hoy los padres están en Milán, apoyando al doctor Dario Miedico, enésimo chivo expiatorio de esta guerra conducida a favor de "la vacuna a toda costa". [leri lo Stato ha deliberatamente abbandonato i genitori che hanno scelto con coscienza per la salute dei propri figli, proponendo un decreto che mira alla ghettizzazione e all'impoverimento delle famiglie, inneggiando a finte epidemie, ricatti e richiami all'Ordine. Oggi i genitori sono a Milano, a sostenere il Dottor Dario Miedico, I'ennesimo capro espiatorio di questa guerra condotta a favore del "vaccino a tutti i costi.] (Página de Facebook Associazione Comilva) ${ }^{(57)}$

He hablado de ultra información y atención excesiva, refiriéndome al concepto de "intensive mothering"(58): la reticencia no se define como un fenómeno claro y nítido ya que representa un proceso constante de negociación entre las propias creencias, necesidades y experiencias. Además, los movimientos italianos se caracterizan también por una configuración dicotómica entre natura/cultura, en la que la vacuna representaría una técnica arriesgada porque es artificial, mientras que la inmunidad adquirida a través de la enfermedad se determinaría como una buena práctica de crear anticuerpos.
Al nacer, el niño está protegido por los anticuerpos trasmitidos directamente de la madre, en caso de que esta esté inmunizada de forma natural (una madre vacunada de niña no transmite anticuerpos a su hijo [...]. En el momento en que la inmunidad desaparece, después de los primeros años de vida, el niño con sistema inmunitario íntegro y saludable puede contraer la enfermedad exantemática con un curso favorable. A partir de ese momento, el niño mantendrá una inmunidad de por vida. [Il bambino, quando nasce, è protetto dagli anticorpi passati ad esso direttamente dalla madre, se immunizzata naturalmente durante la sua infanzia (una madre vaccinata da piccola non trasmette anticorpi al proprio figlio...). Quando questa immunità scompare, dopo i primi anni di vita, il bambino con sistema immunitario integro e con una buona salute generale, può contrarre la malattia esantematica con un decorso favorevole. II bambino, da quel momento conserverà una immunità per tutta la vita.] (Página de Facebook VacciniBasta) ${ }^{(59)}$

Ahora bien, no cabe ninguna duda de que el despliegue de Internet haya favorecido la difusión y expansión de los movimientos de reticencia: las redes sociales han permitido la creación de nuevas identidades virtuales y reales, así como la consolidación de dichos grupos y de sus fuerzas reivindicativas. A pesar de que los contenidos "antivacunas" puedan claramente influir en la percepción del riesgo y de las elecciones en el tema, cabe destacar el papel que juegan las experiencias y sesgos cognitivos en la consolidación de creencias que se ponen en contraste con las narrativas médicas.

En su investigación, Raffaetà subraya la necesidad de algunos padres de recibir más informaciones sobre el tema de vacunas debido a conversaciones contrastantes, poco claras o creíbles por parte de los mé$\operatorname{dicos}^{(55)}$; sin embargo, cabe subrayar la exigencia existencial de todos aquellos padres con hijos autistas que a menudo encuentran 
en la correlación entre autismo y vacuna una manera de comprender las causas de un síndrome del que aún no se conocen todos los factores desencadenantes.

Dichos progenitores refuerzan esta retórica a través de las numerosas informaciones en línea, del intercambio de ideas con los demás padres y del apoyo que reciben de los movimientos de reticencia a la vacunación. Además, hay que añadir que el Estado italiano aplica por ley la indemnización por daño de vacuna, y que existen sentencias jurídicas que reconocen un nexo "altamente probable" entre enfermedad o síndrome y vacunación ${ }^{(60,61,62)}$, por lo que los movimientos de reticencia piden que el tabú sobre las reacciones adversas de las vacunas sea desvelado por parte de la comunidad científica.

En definitiva, la reticencia a la vacunación es un fenómeno social que se conecta de manera indisoluble con la relación entre biomedicina, Estado y comunicación en el contexto del neoliberalismo, hecho que, si ya era importante, más lo es a partir de la crisis del coronavirus, cuya gestión a nivel sanitario, político y económico ha puesto de manifiesto la bien conocida cuestión sobre la comunicación de la ciencia y sus relaciones de poder con el conjunto poblacional. Con "relaciones de poder" me refiero al vínculo entre la producción científica, la política y sus efectos en la población, en las que cabe destacar la gestión de la información y el saber, como reflejo de la relación médico/paciente y Estado/ciudadano en todos sus matices. El papel hegemónico que juega la biomedicina siempre se interconectará con tendencias contrahegemónicas: el fenómeno de la reticencia a la vacunación solo representa una de las múltiples producciones históricas y sociales en las que la ciudadanía sigue revindicando su libertad de elección y autonomía, es decir, su específica concepción de ser-enel-mundo y presencia existencial.

Por supuesto, el primer medio de difusión de dichas tendencias contrahegemónicas son los medios de comunicación masiva $y$, en este contexto, las redes sociales, en las que los fenómenos de filter bubles -es decir, información filtrada- y echochambers -literalmente "caja de resonancia" mediática, que determina un número delimitado de contenidos y la consiguiente amplificación de creencias específicas ${ }^{(63)}$ - resultan ser un factor impactante en la producción o incremento de ideologías.

La elevada cantidad de contenidos en línea, gurús de la información y la presencia de un abanico impactante de páginas, grupos, fórums y blogs han conllevado al hecho de que la competición mediática juegue un papel fundamental en la elección de un lenguaje y de contenidos persuasivos que puedan Ilamar la atención pero que, a nivel consecuencial, podrían conllevar el endurecimiento de las posiciones entre quienes manifiestan creencias y modalidades de pensamiento diferentes. Como se ha visto con el Covid-19, el tema de la comunicación de la ciencia, de su gestión política, y del lugar que deben ocupar la ciencia y la política en la sociedad se han convertido en temas clave en los que se juegan las hegemonías y contrahegemonías de distintos discursos culturales.

Sin embargo, tanto la misma biomedicina como la clase dirigente se caracterizan por ideologismos: la evidencia científica se determina como un saber cristalizado que no presupone dudas ni incertidumbres, al igual que las políticas de salud pública. Las medidas de contención de la reticencia a través de la obligatoriedad de las vacunas y sanciones administrativas son actitudes que no tienen el objetivo de informar a la población, sino de convencerla de que se vacune; hecho por el que, en este clima candente de polarización de la realidad, la relación entre ciencia, política y población necesita una reformulación en la gestión de la comunicación a través de la cual consolidar el sentido de eficacia real y simbólica de la biomedicina en progenitores dudosos o reticentes.

\section{CONCLUSIONES}

En Italia, la reticencia a la vacunación es un fenómeno complejo que se determina por un alto grado de escepticismo no solo en la 
población, sino también en la clase médica y política. El tema de las vacunas se ha convertido en un lugar simbólico, en el que se reflejan las contradicciones y las cuestiones espinosas que forman parte del vínculo entre Estado, institución médica y sociedad. El análisis del contenido compartido en las páginas de Facebook de Associazione Comilva, Movimento 3V, SìAmo y VacciniBasta se ha focalizado principalmente en la correlación entre la vacuna antisarampión y el autismo, siendo esto uno de los argumentos principales más utilizados por los grupos de reticencia para manifestar su objeción de conciencia a la obligatoriedad de las vacunaciones infantiles y más cuestionado por la comunidad científica para desacreditar las ideas "complotistas" de los movimientos antivacunas.

La investigación reveló que la correlación entre vacuna contra el sarampión y autismo se caracteriza fundamentalmente por una reinterpretación de la percepción del riesgo y la evidencia científica: los grupos de reticencia suelen representar la vacuna como un elemento "externo", dañino, que puede afectar gravemente la salud de los infantes ya que contiene elevadas cantidades de metales pesados. De hecho, estos movimientos afirman que, en los niños más vulnerables, la administración de dicha vacuna puede conllevar daños permanentes como autismo. Por estas razones, piden que la obligatoriedad de las vacunaciones infantiles se suspenda, que se hagan pruebas prevacuna y se comercialicen vacunas monodosis, entre otras peticiones. Además, los discursos principales detectados entre las comunidades virtuales se caracterizan también por una configuración dicotómica entre natura/cultura y buenas/malas prácticas de cuidado infantil, así como por una reinterpretación de la responsabilidad individual a través de nuevas figuras parentales atentas y "ultrainformadas".

En el contexto de las vacunaciones infantiles, esta "ultrainformación" se determina como un fenómeno que influye de manera impactante en la percepción del riesgo. Sin embargo, la elevada cantidad de contenido compartido y la elección de un lenguaje persuasivo son factores utilizados tanto por los movimientos de reticencia como por aquellas realidades que apoyan el discurso médico hegemónico. El análisis del contenido compartido en la página de Facebook Medical Facts del médico Roberto Burioni y de las campañas en línea de prevención a la vacunación infantil (véase Figura 1 y Figura 2) revela cómo su comunicación no se destaca de la retórica utilizada por esos mismos movimientos considerados peligrosos y dañinos (véase Figura 3). Esta competición mediática se determina como un factor adicional en la polarización de la realidad, ya que puede amplificar e incrementar determinadas creencias e ideologías opuestas entre ellas. Además, concebir la reticencia a las vacunaciones como una consecuencia de la difusión de fake news en línea podría ser una idea bastante reduccionista y contraproducente: si bien la obligatoriedad ha conllevado un aumento en las tasas vacunales, la aplicación de estrategias de carácter punitivo como las sanciones administrativas y la exclusión del registro de aquellos médicos que expresan ideas "no vax" podría endurecer las posiciones y aumentar la desconfianza en la clase política y la medicina.

Tal vez, las instituciones deberían focalizar la atención en los fenómenos que están en la base de dicha desinformación y en un tipo de comunicación que pueda realmente incidir en la eficacia de la medicina y la relación entre Estado, ciencia y ciudadanos. Como subraya O'Malley, esto "no significa priorizar un conocimiento sobre el otro, sino establecer negociaciones entre ellos: cada uno tiene algo que ofrecer ${ }^{\prime \prime(64)}$.

\section{Limitaciones y aportaciones}

Si bien se reconoce la importancia de realizar investigaciones en línea sobre la reticencia a la vacunación, ya que se trata de movimientos que han adquirido cohesión y popularidad a través de las redes sociales, una comprensión total del fenómeno presupondría una investigación virtual tanto como "real": creo que es indispensable analizar la reticencia bajo sus múltiples perspectivas, es decir, conducir 
investigaciones entre progenitores reticentes y profesionales de la salud de las cuales puedan emerger los factores que inciden en la percepción del riesgo sobre vacunas, como la relación entre médico y paciente, experiencias previas y creencias personales. Sin embargo, investigar las diferentes modalidades de gestión y manejo del riesgo por parte de las instituciones y grupos de reticencia podría aportar sugerencias para futuras estrategias de salud pública tanto a nivel nacional como internacional. Además, la metodología de dicha investigación podría bien aplicarse a otras cuestiones fundamentales como la percepción y el manejo del riesgo durante alarmas sanitarias como la pandemia actual de Coronavirus.

\section{AGRADECIMIENTOS}

Agradezco al doctor Oriol Romaní por haber tutorado la tesis, a la doctora Lina Masana y al doctor Fernando Vidal por el trabajo realizado en calidad de miembros del tribunal. La investigación no presenta algún tipo de conflicto de interés real o potencial.

\section{REFERENCIAS BIBLIOGRÁFICAS}

1. Menéndez E. Las instituciones y sus críticos o la costumbre de polarizar la realidad: el caso de la influenza A (H1N1). Salud Colectiva. 2014;10(1):15-40. doi: $10.18294 /$ sc. 2014.206

2. Genitori Del NO Obbligo Lombardia. Reazioni avverse ai vaccini, la testimonianza di alcuni genitori [Video en Internet]. 2017 [citado 10 jun 2020]. Disponible en: https://tinyurl.com/yasvt6a6.

3. Gruppo Genitori del NO Lombardia [Internet] 2020 [citado 10 jun 2020]. Disponible en: https://tinyurl.com/ yb6hy36c.

4. Italia, Ministero della Salute. Decreto-legge 07, Disposizioni urgenti in materia di prevenzione vaccinale (17G00095) [Internet]. 2017 [citado 10 jun 2020]. Disponible en: https://tinyurl.com/ydbdfthm.

5. Giambi C, Fabiani M, D'Ancona F, Ferrara L, Fiacchini D, Gallo T, Martinelli D, Pascucci MG, Prato R, Filia A, Bella A, Del Manso M, Rizzo C, Rota MC. Parental vaccine hesitancy in Italy - Results from a national survey. Vaccine, 2018;36(6):779-787. doi: 10.1016/j. vaccine.2017.12.074

6. D'Ancona F, D'Amario C, Maraglino F, Rezza G, Ricciardi W, lannazzo S. Introduction of new and reinforcement of existing compulsory vaccinations in Italy: First evaluation of the impact on vaccination coverage in 2017. Eurosurveillance. 2018;23(22):pii $=1800238$. doi: 10.2807/1560-7917.ES.2018.23.22.1800238.

7. Ferro A, Colucci M, Miotto S, Siddu A, Tozzi A. Le caratteristiche dei movimenti antivaccinali. Vaccinare Oggi \& Domani. 2013;4(3):65-68

8. Iorio E. Vaccini e paure: Salute pubblica, resistenze popolari. Venetica. 2018;(54):15-36.
9. Johannessen $\mathrm{H}$, Lazar I. Multiple medical realities: Patients and healers in biomedical, alternative and traditional medicine. New York: Berghahm Books; 2006.

10. Perdiguero E. El fenómeno del pluralismo asistencial: una realidad por investigar. Gaceta Sanitaria. 2004;18(Supl 1):140-145.

11. Riccò I. En busca de un «nuevo mundo mágico»: De la medicina popular a las terapias New Age en un Occidente desencantado. [Tesis de doctorado]. Tarragona: Universitat Rovira i Virgili; 2017.

12. Raffaetà R. When 'to choose' is 'to care': the case of paediatric vaccinations. Suomen Antropologi: Journal of the Finnish Anthropological Society. 2012;37(3):8-23.

13. Ardevol E, Estalella A. Internet: instrumento de investigación y campo de estudio para la antropología visual. Revista Chilena de Antropología Visual. 2010;15:1-21.

14. Dalsgaard S. The ethnographic use of Facebook in everyday life. Anthropological Forum. 2016;26(1):96114. doi: 10.1080/00664677.2016.1148011.

15. Hart T. Online Ethnography. En: Matthes J, Davis CS, Potter RF, (ed). The International Encyclopedia of Communication Research Methods. Wiley; 2017. doi: 10.1002/9781118901731.iecrm0172.

16. Menéndez E. Modelo hegemónico, modelo alternativo subordinado, modelo de autoatención: caracteres estructurales. En: La antropología médica en México. México: Universidad Autónoma Metropolitana; 1982. p. 97-113.

17. Foucault M. Historia de la medicalización. En: Historia de los hombres infames: Ensayos sobre desviación y dominación. Madrid: Ediciones La Piqueta; 1992. p. 122-152.

18. Beck U. La sociedad de riesgo: Hacia una nueva modernidad. Barcelona: Paidós; 1998.

19. Sepúlveda Galeas M. Gubernamentalidad y riesgo en el campo de las drogas: cuando la chance deviene presagio. El Cotidiano. 2010;(163):55-65.

20. Sepúlveda Galeas M, Romaní O. Conceptualización y políticas de la gestión del riesgo. En: Martínez Oró DP, 
Pallarés Gómez J, (eds.). De riesgos y placeres: Manual para entender las drogas. Lleida: Milenio; 2013. p. 89102.

21. Romaní O. Reducción de daños y control social: ¿¿De qué estamos hablando? En: Martínez Oró DP, Pallarés Gómez J, (eds.). De riesgos y placeres: Manual para entender las drogas. Lleida: Milenio; 2013. p. 103-116.

22. Lupton D. Risk as moral danger: The social and political functions of risk discourse in public health. International Journal of Health Services. 1993;23(3):425-435. doi: 10.2190/16AY-E2GC-DFLD-51X2.

23. Douglas M. La stregoneria: Confessioni e accuse, nell'analisi di storici e antropologi. Torino: Einaudi; 1980.

24. Douglas M. Risk as a forensic resource. Daedalus. 1990;119(4):1-16.

25. Romaní O. La reducció de riscos, entre l'experiència i els experts. Quadernes-E de L'Ica. 2013;18(1):52-64.

26. Nichter M. Reducción del daño: una preocupación central para la antropología médica. Desacatos. 2006;20:109-132.

27. Kahan DM. A risky science communication environment for vaccines. Science. 2013;342(6154):53-54. doi: 10.1126/science.1245724.

28. Vaccini Basta. Liberi Dai Vacciini tramite Autismo\&Vaccini [Internet]. 28 abr 2013 [citado 10 jun 2020]. Disponible en: https://tinyurl.com/yb2fd2ve.

29. Comilva. I vaccini non causano l'autismo... [Internet]. 10 sep 2015 [citado 10 jun 2020]. Disponible en: https://tinyurl.com/yc7mxsmt.

30. Associazione Nazionale Genitori perSone con Autismo. Autismo [Internet]. 2020 [citado 10 jun 2020]. Disponible en: http://angsa.it/autismo/

31. Associazione Comilva. Grandissimo intervento del sen. Maurizio Romani poco fa in diretta dal senato [Internet]. 12 jul 2017 [citado 10 jun 2020]. Disponible en: https://tinyurl.com/y854vs48.

32. Comilva. Sostieni I'informazione indipendente [Internet]. 27 feb 2018 [citado 10 jun 2020]. Disponible en: https://tinyurl.com/yckxlx7s.

33. Comilva. I vaccini non causano l'autismo... [Internet]. 10 sep 2015 [citado 10 jun 2020]. Disponible en: https://tinyurl.com/yc7mxsmt.

34. Pezzotti $P$, Bellino S, Prestinaci $F$, lacchini S, Lucaroni F, Camoni L, Barbieri MM, Ricciardi W, Stefanelli P, Rezza G. The impact of immunization programs on 10 vaccine preventable diseases in Italy: 1900-2015. Vaccine. 2018;36(11):1435-1443. doi: 10.1016/j.vaccine.2018.01.065.

35. Gualano MR, Bert F, Voglino G, Buttinelli E, D'Errico MM, De Waure C, Di Giovanni P, Fantini MP, Giuliani AR, Marranzano M, Masanotti G, Massimi A, Nante N,
Pennino F, Squeri R, Stefanati A, Signorelli C, Siliquini R, Castaldi S, Zappalà G. Attitudes towards compulsory vaccination in Italy: Results from the NAVIDAD multicentre study. Vaccine. 2018;36(23):3368-3374. doi: 10.1016/j.vaccine.2018.04.029.

36. Anello $\mathrm{P}$, Cestari L, Baldovin $\mathrm{T}$, Simonato $\mathrm{L}$, Frasca G, Caranci N, Grazia Pascucci M, Valent F, Canova C. Socioeconomic factors influencing childhood vaccination in two northern Italian regions. Vaccine. 2017;35(36):4673-4680. doi: 10.1016/j.vaccine.2017.07.058.

37. Wolfe RM, Sharp LK. Anti-vaccinationists past and present. BMJ. 2002;325:430-432. doi: 10.1136/ bmj.325.7361.430.

38. Poland GA, Jacobson RM. Understanding those who do not understand: A brief review of the anti-vaccine movement. Vaccine. 2001:19(17-19):2440-2445. doi: 10.1016/S0264-410X(00)00469-2.

39. Associazione Comilva. Lawrence Solomon: QUELLO CHE DEL MORBILLO NON VIENE DETTO [Internet]. 19 abr 2014 [citado 10 jun 2020]. Disponible en: https:// tinyurl.com/yd5jmgsh.

40. Vaccini Basta. è uscito il nuovo bollettino del morbillo [Internet]. 15 sep 2017 [citado 10 jun 2020]. Disponible en: https://tinyurl.com/yczc3wml.

41. SìAmo. La giornata della consapevolezza sull'autismo [Internet]. 2 abr 2019 [citado 10 jun 2020]. Disponible en: https://tinyurl.com/y6ugtthp.

42. Comilva. Si dissolvono le illusioni sul vaccino antimorbillo [Internet]. 18 mar 2016 [citado 10 jun 2020]. Disponible en: https://tinyurl.com/y8axy4zf.

43. Matteo Salvini. Anche dall'opposizione, grande successo della Lega! [Internet]. 29 jul 2017 [citado 10 jun 2020]. Disponible en: https://tinyurl.com/ybtz3vry.

44. MoVimento 5 Stelle. Come abbiamo sempre detto, riteniamo i vaccini fondamentali nella prevenzione delle malattie... [Internet]. 26 jul 2017 [citado 10 jun 2020]. Disponible en: https://tinyurl.com/yctr9eys.

45. Movimento 3V Vaccini Vogliamo Verità. Elezioni Regionali Emilia Romagna [Internet]. 13 jun 2020 [citado 10 jun 2020]. Disponible en: https://tinyurl.com/ y8bw86j4.

46. Movimento 3V. Un giorno, un crudele sovrano impose l'obbligo a tutto il regno [Internet]. 14 jun 2020 [citado 10 jun 2020]. Disponible en: https://tinyurl.com/ ydx3cf3a.

47. Medical Facts di Roberto Burioni. Información Medical Facts di Roberto Burioni. Información [Internet]. 14 jun 2020 [citado 14 jun 2020]. Disponible en: https:// tinyurl.com/ybuk5azq.

48. Medical Facts di Roberto Burioni. La diffusione di bugie pericolose, soprattutto quando si parla di salute, è una cosa gravissima... [Internet]. 28 jul 2018 [citado 14 jun 2020]. Disponible en: https://tinyurl.com/y96xgkzj. 
49. Medical Facts di Roberto Burioni. Ecco come questo scienziato italiano è divenuto una celebrità contrastando gli scettici dei vaccini [Internet]. 28 jul 2018 [citado 14 jun 2020]. Disponible en: https://tinyurl.com/y7ygp8kb.

50. Medical Facts di Roberto Burioni. aDobbiamo tutelare il diritto alla salute e alla istruzione dei bambini che non si possono vaccinare... [Internet]. 19 may 2018 [citado 14 jun 2020]. Disponible en: https://tinyurl.com/ ybevdgzh.

51. Medical Facts di Roberto Burioni. La scienza ci dice con certezza che i vaccini sono sicuri ed efficaci... [Internet]. 28 feb 2017 [citado 14 jun 2020]. Disponible en: https://tinyurl.com/yc2u9duo.

52. Bocci M. Treviso: I'Ordine radia Gava, il medico antivaccini. La Repubblica [Internet]. 21 abr 2017 [citado 14 jun 2020]. Disponible en: https://tinyurl.com/ y7c2ksro.

53. La Repubblica. Milano, medico no-vax radiato dall'Ordine. Burioni: "Buona notizia" [Internet]. 12 abr 2018 [citado 14 jun 2020]. Disponible en: https:// tinyurl.com/y986kfeg.

54. Nuñez M. Mark Zuckerberg's Answer To An AntiVaxxer Question Highlights Facebook's Problematic Response To Misinformation [Internet]. 23 oct 2019 [citado 14 jun 2020]. Disponible en: https://tinyurl.com/ y7txvqha.

55. Raffaetà R. Biosociality extended: The case of parental groups campaigning against paediatric vaccinations in Italy. Emerging Socialities in 21st Century Healthcare, Amsterdam University Press 2017;13-24.

56. Associazione Comilva. Ecco un libro adatto a tutti genitori, a prescindere dalle scelte in ambito vaccinale... [Internet]. 19 jun 2017 [citado 14 jun 2020]. Disponible en: https://tinyurl.com/y8wsvht7.
57. Associazione Comilva. Ieri lo Stato ha deliberatamente abbandonato i genitori che hanno scelto con coscienza per la salute dei propri figli... [Internet]. 20 may 2017 [citado 14 jun 2020]. Disponible en: https://tinyurl. com/ya5386pv.

58. Reich JA. Neoliberal Mothering and Vaccine Refusal: Imagined Gated Communities and the Privilege of Choice. Gender and Society. 2014;28(5):679-704. doi: $10.1177 / 0891243214532711$

59. Vaccini Basta. MORBILLO: Dividiamo il foglio in due parti. Da una parte analizziamo il processo di immunizzazione naturale e ciò che ne deriva [Internet]. 24 jun 2017 [citado 14 jun 2020]. Disponible en: https:// tinyurl.com/y9fah9gc.

60. Comilva. Sentenze danno da vaccino [Internet]. 7 jul 2013 [citado 10 febr 2021]. Disponible en: http://tinyurl. com/5q45merf.

61. II Fatto Quotidiano. Modena, "neonato disabile per vaccino": il Tar impone al ministero della Salute risarcimento per "danni da esavalente" [Internet]. 28 feb 2019 [citado 14 jun 2020]. Disponible en: https://tinyurl.com/ ydyaqtaf.

62. La Stampa. Una sentenza riconosce indennizzo per danni da vaccino [Internet]. 5 jun 2017 [citado 14 jun 2020]. Disponible en: https://tinyurl.com/ycxkwy4j.

63. Damele G. L'impatto dei social media sulla cittadinanza politica. En: Seminari su La Tecnologia e la Persona Ancona. Roma: Pontificio Ateneo Sant'Anselmo; 2019.

64. O' Malley P. Experimentos en gobierno: Analíticas gubernamentales y conocimiento estratégico del riesgo. Revista Argentina de Sociología. 2007;(8):151-171.

\section{FORMA DE CITAR}

"Si hay un riesgo, quiero poder elegir": gestión y percepción del riesgo en los movimientos de reticencia a la vacunación italianos. Salud Colectiva. 2021;17:e3231. doi: 10.18294/sc.2021.3231

Recibido: 9 oct 2020 | Versión final: 13 nov 2020 | Aprobado: 18 nov 2020 | Publicado en línea: 10 feb 2021 